\title{
Individual consistency in the behaviors of newly-settled reef fish
}

James R White, Mark G Meekan, Mark I McCormick

Flexibility in behavior is advantageous for organisms that transition between stages of a complex life history. However, various constraints can set limits on plasticity, giving rise to the existence of personalities that have associated costs and benefits. Here, we document a field and laboratory experiment that examines the consistency of measures of boldness, activity, and aggressive behavior in the young of a tropical reef fish, Pomacentrus

amboinensis (Pomacentridae) immediately following their transition between pelagic larval and benthic juvenile habitats. Newly-settled fish were observed in aquaria and in the field on replicated patches of natural habitat cleared of resident fishes. Seven behavioral traits representing aspects of boldness, activity and aggression were monitored directly and via video camera over short (minutes), medium (hours), and long (3 days) time scales. With the exception of aggression, these behaviors were found to be moderately or highly consistent over all time scales in both laboratory and field settings, implying that these fish show stable personalities within various settings. Our study is the first to examine the temporal constancy of behaviors in both field and laboratory settings in over various time scales at a critically important phase during the life cycle of a reef fish. 
3

4

5

6

19 Contact:

20 James White

21 College of Marine and Environmental Sciences

22 James Cook University

23 Townsville, QLD 4811

24 Australia

25 james.ryan.white@gmail.com

$26 \quad \mathrm{P}:+610408801682$

$27 \quad F:+61747251570$
James R. White ${ }^{1,2}$, Mark G. Meekan³, Mark I. McCormick ${ }^{1,2}$

${ }^{1}$ College of Marine and Environmental Sciences, James Cook University, Townsville, Queensland, Australia ${ }^{2} A R C$ Centre of Excellence for Coral Reef Studies, James Cook University, Townsville, Queensland, Australia

Australia 
29 There has been considerable interest in, and evidence for, consistent patterns in the behaviors of individual

30 animals within a species over the last decade (Dall, Houston \& McNamara, 2004; Sih, Bell \& Johnson, 2004; Sih

31 et al., 2004; Dingemanse \& Réale, 2005; Bell, 2007; Réale et al., 2007; Smith \& Blumstein, 2008; Bell, Hankison

32 \& Laskowski, 2009). Differences in the amount of aggressive, exploratory and bold behaviors among individuals

33 have been shown to be widespread and heritable (Boake, 1994; Stirling, Reale \& Roff, 2002; Kolliker, 2005; van

34 Oers et al., 2005; Réale et al., 2007) across a diverse array of taxa (Dingemanse \& Réale, 2005; Smith \&

35 Blumstein, 2008) and to influence survival (Downes, 2002; Dingemanse et al., 2004), reproductive success

36 (Both et al., 2005; Sih \& Watters, 2005; Pruitt \& Ferrari, 2011), resource acquisition (Webster, Ward \& Hart,

37 2009) and growth. Adopting a certain behavioral phenotype can have both costs and benefits, for example,

38 highly aggressive female fishing spiders (Dolomedes triton) are more successful at acquiring food in a

39 competitive environment, but this aggression can be detrimental in another context such as when it leads to

40 precopulatory sexual cannibalism (Johnson \& Sih, 2005). Thus, consistent patterns in behavior among

41 individuals can lead to trade-offs in aspects of fitness, which can ultimately influence population dynamics,

42 community structure, and species diversity (Pruitt, Grinsted \& Settepani, 2013; Mittelbach et al., 2014).

43 Variation in consistent behavioral patterns among individuals have been variously (and interchangeably)

44 termed 'behavioral syndromes', 'temperament', 'personality', and 'coping styles' (Dall, Houston \& McNamara,

45 2004; Réale et al., 2007; Dingemanse et al., 2010; Sih et al., 2012), although some authors have argued for a

46 more restrictive use of terminology (Bell, Hankison \& Laskowski, 2009; Garamszegi \& Herczeg, 2012). Here, we

47 adopt the definitions of Garamszegi and Herczeg (2012), where consistency in single behaviors (e.g. individuals

48 that display repeatedly higher or lower levels of boldness, exploration, or aggression than others in the

49 population) are described as displaying 'personality', and consistency in the relationship between two or more

50 functionally different behaviors within the same individual is defined as a 'behavioral syndrome'. For example,

51 a behavioral syndrome is evident in the correlation between boldness and aggression documented within 
52 individual sticklebacks (Gasterosteus aculeatus) (Bell, 2005) and funnel-web spiders (Agelenopsis aperta)

53 (Riechert \& Hedrick, 1993).

54 Although the ability to alter behavior to suit changing environmental conditions is likely to be advantageous

55 (Kelley, Phillips \& Evans, 2013), behavior is not infinitely plastic (DeWitt, Sih \& Wilson, 1998). If a single optimal

56 behavioral phenotype existed, natural selection should reduce genotypic variation over generations (Réale et

57 al., 2007). Because behavioral phenotypes show heritable variation not eroded by selection (Penke, Denissen \&

58 Miller, 2007; Réale et al., 2007), different behavioral strategies are likely to have different associated costs and

59 benefits (Kelley, Phillips \& Evans, 2013). For example, larger, bolder and faster-growing phenotypes of rainbow

60 trout (Oncorhynchus mykiss) are more likely to be captured by fishing gears (Biro \& Post, 2008).

61 Estimating the consistency of a behavioral trait is necessary for measuring the repeatable characteristics of a

62 focal organism, quantifying trait plasticity and determining trait heritability (Nakagawa et al., 2007).

63 Historically, personality studies using a single assay were common, but it has been recently suggested that

64 repeated tests are essential for any personality study (Réale et al., 2007) and the strength of behavioral

65 syndromes are likely underestimated when based upon single assays of varying traits (Adolph \& Hardin, 2007;

66 Beckmann \& Biro, 2013; White et al., 2013; White, McCormick \& Meekan, 2013).

67 Clearly, there is a need to determine the consistency of behaviors before examinations of personality,

68 behavioral syndromes and associated trade-offs of alternative behavioral strategies can be attempted. Here,

69 we examine evidence for personalities in a juvenile tropical reef fish, the Ambon damselfish (Pomacentrus

70 amboinensis), by establishing the consistency of commonly-used field and laboratory assays of activity,

71 aggression and boldness over time scales ranging from minutes to days following settlement. Similar to many

72 reef fishes, young of this species can be collected at the end of their larval phase immediately prior to

73 settlement on the reef, when they are naïve to reef-based predators and behaviors learned after settlement

74 (Meekan et al., 2010). In this immediate post-settlement phase of their life cycle, reef fishes typically 
experience very high mortality (Almany \& Webster, 2006), with rates within the first 48 hours of benthic life averaging 57\% (Doherty et al., 2004; Almany \& Webster, 2006). Because experience can influence behavioral phenotypes (Budaev, 1997; Bell \& Sih, 2007; Dingemanse et al., 2009), the use of naïve study organisms allows us to control for variation and consistency in behavior associated with experience and to examine ecologically important behavioral traits at a critical ontogenetic boundary (McCormick \& Meekan, 2010; Poulos \& McCormick, 2014). Because field measurements are made directly by an observer on SCUBA (where visual and auditory presence is not easily concealed), we tested for an effect of observer presence by comparing observed behaviors to those recorded by video-camera. Specifically, we aimed to determine if juvenile damselfish behaviors were: 1) significantly altered by observer presence; 2 ) consistent over various time scales (minutes, hours, days) relevant to their major mortality bottleneck (first 48 hours following settlement); 3) consistent in an aquarium setting; and 4) correlated between field and lab-based measurements. Based on our anecdotal previous experience with this system and study species, we predicted all behaviors to be moderately consistent in the field and laboratory.

\section{Methods}

\section{Ethics statement}

Fish collection locations/activities and handling protocols were approved by the Great Barrier Reef Marine Park Authority (Permit Number: G10/33784.1) and JCU Animal Ethics Committee (Permit Number: A1720). All efforts were made to minimize animal handling and stress.

\section{Study site and species}

This study was conducted on the shallow reef (2-4 m depth) offshore from the Lizard Island Research Station $\left(14^{\circ} 40^{\prime} \mathrm{S}, 145^{\circ} 28^{\prime} \mathrm{E}\right)$ on the northern Great Barrier Reef, Australia. Our study species, the Ambon damsel, $P$. amboinensis, is common on Indo-Pacific coral reefs (Beukers \& Jones, 1998). After approximately 20 days as pelagic larvae and at about $11 \mathrm{~mm}$ standard length (Wellington \& Victor, 1989), young fish settle from the 
98 plankton at night to reefs (Pitcher, 1988). These fish preferentially choose to settle on live coral (McCormick \&

99 Weaver, 2012) and settlement occurs predominantly between October and January around the time of the

100 new moon (Meekan, Milicich \& Doherty, 1993). Newly settled fish are found as solitary individuals associated

101 with conspecific adults and sub-adults (McCormick \& Makey, 1997). P. amboinensis has a relatively small home

102 range (Brunton \& Booth, 2003), moving only small distances $(<1 \mathrm{~m})$ during the first few months after

103 settlement (McCormick \& Makey, 1997). Due to its high abundance, small size, rapid development, and

104 sedentary nature, $P$. amboinensis is an ideal model organism for field and laboratory based behavioral studies

105 (Meekan et al., 2010).

106 Experimental design

107 Collection

108 We collected newly-metamorphosed juveniles of $P$. amboinensis (McCormick \& Makey, 1997) using moored

109 light traps (see small light trap of Figure 1 in Meekan et al. 2001 for design) during the October recruitment

110 pulse. Different cohorts of fish were used for the different experiments. Traps were anchored approximately

$111100 \mathrm{~m}$ from the nearest reef in $10 \mathrm{~m}$ of water at dusk and left overnight. Catches were emptied from the traps

112 the next morning between 05:30-07:00 h. All fish collected from the traps were transported to the laboratory

113 where $P$. amboinensis was separated from all other species and maintained in a $25 \mathrm{~L}$ aquarium (at densities $<$

114100 individuals $/ 25 \mathrm{~L}$ ) of aerated seawater for $24 \mathrm{~h}$ to acclimatize to local conditions and reduce handling stress

115 before experiments began. Fish were fed Artemia nauplii twice daily while in captivity. For field experiments,

116 each acclimated P. amboinensis was transported to the field in individually-labeled clip-seal plastic bag. After

117 final observations, study organisms were released unharmed on nearby natural habitat.

118 Observational protocol

119 Behavioral consistency in the field 
120 All behavioral observations were made on individual fish in the field or aquaria in the laboratory using separate

121 groups of fish for each assessment. Each P. amboinensis was placed into a labeled $2 \mathrm{~L}$ clip-seal plastic bag

122 containing aerated seawater and transported to the field. Divers released an individual fish onto a small patch

123 reef $(30 \times 30 \times 30 \mathrm{~cm})$ constructed from live and dead pieces of the bushy hard coral Pocillopora damicornis on

124 the shallow (3-4 m water depth) sand flat. P. amboinensis recruits occur naturally in this habitat. Reefs were

125 deployed in a single row, approximately $3 \mathrm{~m}$ apart, parallel to and $5 \mathrm{~m}$ from the nearest area of natural reef.

126 Means and ranges of temperatures did not vary among reefs or among aquaria (M. McCormick unpubl. data)

127 and care was taken in reef construction to ensure that patch reefs had only very minor differences in habitat

128 structure. Previous studies have shown that such minor variation in topographic complexity of patch reefs has

129 no effect on behavior of young fish (McCormick \& Meekan, 2010; Meekan et al., 2010). Before introduction of

130 the study fish, patch reefs were cleared of any resident fishes using hand nets. These were released on nearby

131 natural reef far enough away to prevent their return (approx. $10 \mathrm{~m}$ ). Individual study fish were then released

132 onto their respective patch reefs and the first behavioral variable (latency to enter a novel environment; see

133 description below) was recorded. Immediately afterwards, small wire cages (about $30 \times 30 \times 30 \mathrm{~cm}, 12 \mathrm{~mm}$

134 mesh size) were placed over the patch to allow the fish to acclimate to the new surroundings while being

135 protected from predation. Cages were left a minimum of 20 min and carefully removed immediately before

136 observations. Following established protocols outlined below (McCormick \& Meekan, 2010; Meekan et al.,

137 2010; White et al., 2013), divers conducted observations from at least $1 \mathrm{~m}$ away (with the aid of a $2 \mathrm{x}$

138 magnifying glass) to avoid any effects that may have been caused by the proximity of the observer to the

139 target fish.

140 Short term consistency

141 Three behavioral measures of activity were recorded simultaneously over a 3 min observation interval for each

142 fish ( $n=18$ ) during October 2009: bite rate (number of feeding strikes towards objects floating in the water

143 column); distance ventured (DV; the maximum distance in centimeters fish moved away from their patch reef) 
144 and; height on the reef (categorized as a cumulative proportion of the time spent at varying heights over the 3

145 min observation period, with the top of the patch taken as height of 1 , middle of the patch a height of 0.5 , and

146 bottom a height of 0 ). Relative height on the patch was summarized as a cumulative proportion of the time

147 spent at varying heights over the 3 min observation period, calculated from the sum of the proportions

148 multiplied by the height categories (0, 0.5, or 1 ). Following the 3 min interval, a $30 \times 30 \mathrm{~cm}$ acrylic mirror

149 (mounted on a $1 \mathrm{~m}$ PVC pole) was gently placed $10 \mathrm{~cm}$ in front of the focal fish. After a 1 min acclimation

150 period, two scores of aggression were recorded as latency until first strike ('attack latency') and 'mirror strike

151 rate' (combined number of strikes or tail whips) made toward their reflection over 3 min was recorded (Gerlai,

152 2003; Marks et al., 2005). To examine the level of behavioral consistency over a 2 hour period, the entire suite

153 of behavioral assays were repeated three times with 30 min between observations over a single day.

154 Consistency over multiple days in field

155 A separate sample of fish ( $n=21$ ) was used to assess behavior over multiple days in October 2012. Observations

156 were made 3 times each day (at 9:00, 12:00, 16:00 h) for each of 3 days giving a total of 9 repeated

157 observations per individual. During each observation, activity (bite rates, distance ventured (DV), and height)

158 was recorded as described earlier.

159

160

161

162

163

164

165

166

Observer vs. video

To assess if there were any effects of observer presence, behaviors were recorded with a GoPro Hero $2^{\text {TM }}$ high definition video camera (720p resolution) and compared against observer scores $(n=29)$ using fish collected in

October 2012. The camera was placed $30 \mathrm{~cm}$ from focal fish and left to record for $10 \mathrm{~min}$. The first observation was a 3 min period of the behaviors recorded by the observer ( $1 \mathrm{~m}$ away) and camera simultaneously. The second observation was the last recorded 3 min of video (without an observer present). For analysis, this provided three data sets for every fish: 'observer', the 'simultaneous video' recorded at the same time as the direct observation, and the 'video' recording without observer presence. Because of the difficulty in discerning 
167 distance in the video, only bite rates and height (see below) were recorded and observations in which fish

168 moved out of view of the camera for more than $20 \mathrm{sec}$ in total were discarded. Although the recording of

169 observations (observer, simultaneous video and video) in the same order could have potentially introduced a

170 habituation effect, we followed this protocol because it minimized disturbance to fish.

\section{Behavioral consistency in the laboratory}

\section{Short term consistency}

173 Individual fish $(\mathrm{n}=10)$ were assessed for boldness during the 2012 field season using a variation of a common

174 test, latency to emerge from a shelter (Budaev, 1997; Fraser et al., 2001; Brown, Jones \& Braithwaite, 2005;

175 Chang et al., 2012). Each fish was gently transferred via hand net into an opaque $162 \mathrm{~cm}^{3}$ plastic holding

176 chamber within an aquaria (13L, $20 \mathrm{~cm}$ water depth) that also contained a small refuge of live Pocillopora

177 damicornis at the opposite end and allowed to acclimatize for $30 \mathrm{~min}$. The holding chamber was believed to be

178 of adequate size because the fish displayed no apparent signs of confinement stress. The sides of each

179 aquarium were blacked out with plastic sheeting to isolate them from neighboring tanks. After acclimation,

180 observers standing behind a blind (black plastic sheeting) gently revealed the opening to the holding chamber.

181 Time to emerge ('latency to emerge'; defined as more than half of the body length outside of the holding

182 chamber), was recorded for each fish with a cut-off time for the observation of $180 \mathrm{~s}$. Location (categorized as

183 a cumulative proportion of the time spent in various sections of the aquaria, with the third of the aquaria with

184 the chamber given a value of 1 , middle third of the aquaria a value of 0.5 , and the third with coral refuge a

185 value of 0 ) was recorded in the 5 minutes following emergence. A location score was calculated from the sum

186 of the proportions multiplied by the location categories. Here, a lower location score represents a bolder fish.

187 To get to the coral refuge they must exit the chamber and swim across the length of the aquaria, while a shyer

188 fish would not risk leaving the chamber. Aggression was tested by gently placing an acrylic mirror $(30 \times 15 \mathrm{~cm})$

189 upright on the back wall of the aquaria, with the aquaria orientated lengthwise to the observer. Traits of 
aggression were measured in the same manner as in the field, as outlined earlier. Water flow was shut off

191 during the acclimation period and behavioral observations to reduce auditory disturbance, but a gentle air flow

192 through air stones was maintained to ensure adequate dissolved oxygen levels. Fish were fasted for $12 \mathrm{~h}$

193 before trials and fed Artemia upon completion to prevent varying hunger levels of individual fish potentially

194 confounding behaviors. Assays were repeated 3 times over a $2 \mathrm{~h}$ period throughout a single day.

195

196

197

198

199

\section{Field vs. laboratory}

One sample of fish ( $\mathrm{n}=32$ ) was compared across field and aquaria settings in 2012. In the morning (9:00) $P$. amboinensis within $2 \mathrm{~d}$ of capture by light traps were assessed for boldness (latency to emerge and location) and aggression (attack latency and strikes) in aquaria as described above. Later that afternoon (13:00) they were assessed for release latency, bite rate, distance ventured, height, and aggression (attack latency and mirror strike rate) in the field as described earlier. After resident fish were cleared from the patch reefs, each damselfish was carefully released from the plastic bag onto the sand $10 \mathrm{~cm}$ from the patch reef. Latency to emerge was the amount of time it took for the fish to move onto refuge of the patch reef and was timed from the moment the fish exited the bag, to the instant it reached the edge of the reef shelter.

\section{Data analysis}

For all fish (total $n=110$ ), consistency was calculated with a repeatability score $(R)$, defined as the intra-class correlation coefficient (ICC), representing the fraction of total variation in a set of measurements attributable to the variance among individuals (Wolak, Fairbairn \& Paulsen, 2012). R was calculated by constructing a general linear mixed model with individual (ID) included as a random factor in a one-way analysis of variance (ANOVA) model, with the transformed behavioral score as the dependent variable. All scores were $\log _{10}(x+1)$ transformed to meet the assumption of normality and linearity. The ratio of variance explained by amongindividual variance to total variance calculated from an ANOVA represents a common measure of repeatability of each behavior (Lessells \& Boag, 1987). Confidence intervals (CI) around each repeatability estimate were 
213 calculated using the exact confidence limit equation in Searle (1971), which has been shown to be precise for

214 this type of dataset (Donner \& Wells, 1986; Wolak, Fairbairn \& Paulsen, 2012). The R value indicates the

215 strength of repeatability and ranges from 0 to 1, with values approaching one indicating high repeatability

216 (Briffa \& Greenaway, 2011). The p-value associated with the ANOVA is then used to determine if repeatability

217 is significantly greater than zero (Lessells \& Boag, 1987).

218 Relationships between behavioral traits observed in the field and aquaria were analyzed using Pearson's

219 product-moment correlation. All scores were $\log _{10}(x+1)$ transformed to improve normality. Statistical analysis 220 used SPSS version 20.0 (SPSS Inc., Chicago, IL, U.S.A.).

\section{Results}

\section{Short term consistency in the field}

223 In the field, activity measurements (bite rate, DV, and reef height) were highly repeatable, with repeatability

224 scores between 0.52 and $0.69(n=18$, Table 1). The aggression measures (attack latency and mirror strike rate)

225 decreased over time and were not significantly repeatable. By the third observation, fish did not respond to

226 their reflection aggressively at all, suggesting that they became habituated to the mirror.

\section{Consistency over multiple days in field}

228 Fish sampled three times a day for 3 days also displayed activity (bite rate, DV, and height) behaviors that were 229 moderately to highly consistent ( $n=21, R=0.33$ to 0.77 ; Table 1 ).

230 Observer vs. video

231 Observer and simultaneously collected video data were very consistent ( $n=29, R=0.46$ bite rate, 0.76 reef

232 height: Table 1), as were the two video observations ( $n=29, R=0.69$ bite rate, 0.89 reef height; Table 1). 
234 The measure of boldness (i.e., latency to emerge) and location after emergence were moderately consistent

$235(\mathrm{n}=10, \mathrm{R}=0.38$ and 0.54 respectively; Table 1$)$.

\section{Field vs. laboratory}

237 There were only two significant correlations between field and laboratory-based measurements of behavior, 238 with a moderate positive correlation between latency to emerge values in the field and the lab $(n=32, r=0.35$, $239 \mathrm{p}=0.049$; Table 2) and between field and lab measures of aggression latency $(n=32, r=-0.385, p=0.030 ;$ Table 2).

240 The other variables (i.e. measures of location and aggression) showed no evidence of consistency between 241 laboratory and field measurements, suggesting that the behaviors are context dependent and laboratory 242 measures have little relevance to field studies.

\section{Discussion}

244 Our study is one of the most detailed assessments of behavioral consistency of a marine organism to date. It 245 shows that shortly after entering a new habitat at the end of their larval phase fish approximately three weeks 246 old already have a complex repertoire of behaviors that are displayed in a consistent way through time, 247 indicative of the existence of individual personalities. Moreover, this personality appears to be established 248 prior to or immediately upon metamorphosis and settlement. Factors that are likely to favor consistent over 249 conditional behavior, and thus give rise to individual personalities are diverse and include: genetic, 250 physiological or developmental limits, costs of flexibility, costs and availability of information acquisition, 251 metabolism, body size, or constraints on behavioral plasticity (Sih, Bell \& Johnson, 2004; Bergmuller, Schurch \& 252 Hamilton, 2010; Briffa \& Greenaway, 2011). Stable behavioral states are hypothesized to be created when 253 positive feedback loops form between state variables such as size, competitive ability, or condition and state254 dependent behavioral decisions (Dall, Houston \& McNamara, 2004; Sih \& Bell, 2008). For example, individuals 255 with higher body condition may be more cooperative compared to those in poorer condition because they can 256 afford the energy expenditure. If cooperative behavior then led to increased energy gains, this feedback loop 
257 would maintain higher body condition (Bergmuller, Schurch \& Hamilton, 2010). Naïve juvenile reef fish

258 exhibiting personalities at settlement suggests a genetic component and strong trade-offs related to adopting

259 alternative personalities. High mortality rates at this phase of their life cycle could provide very strong selective

260 force and are most likely to be involved (McCormick \& Meekan, 2010).

261 Generally, our study found moderate to highly repeatable behavioral scores for almost all behavioral measures. These ranged from 0.33 (height on the habitat patch across multiple days) to 0.89 (height across camera observations), values well within the range recorded by earlier studies. A recent meta-analysis by Bell et al. (2009) reported an average repeatability value of 0.37 in various behavioral traits across 114 studies and 98 species. They found mating, habitat selection and aggression to be the most repeatable traits; while activity, mate preference, and migration were the least repeatable. Consistency was generally higher for behaviors measured at closer time intervals, juveniles compared to adults and field studies versus laboratory settings (Bell, Hankison \& Laskowski, 2009). Approximately 70\% of this distribution was between 0.1 and 0.6 (see Fig. 1, Bell et al. 2009). An additional 11 studies published more recently (Réale et al., 2000; Smith \& Blumstein, 2008; Briffa \& Greenaway, 2011; Marras et al., 2011; Couchoux \& Cresswell, 2012; Carter et al., 2012; Beckmann \& Biro, 2013; Neumann et al., 2013; Pruitt, Grinsted \& Settepani, 2013; Kelley, Phillips \& Evans, 2013; Burtka \& Grindstaff, 2013) reported repeatability scores ranging from as low as 0.14 for a measure of aggression in male crested macaques (Macaca nigra) (Neumann et al., 2013) to as high as 0.92 for a measure of escape response in European sea bass (Dicentrarchus labrax) (Marras et al., 2011). Despite the wide range in these scores, they were cited as evidence of the consistency of behaviors and therefore personalities. On this basis, the repeatability scores we obtained suggest evidence for personality in the 3-week old damselfish that were the subjects of our study. behavior (Jones \& Godin, 2009). While juvenile damselfish are known to adopt a wide range of behavioral 
281 due to plasticity in the amount of habituation to the experimental protocol (Martin \& Réale, 2008). Across

282 repeated trials, an environment or test may become less novel and individuals may habituate to novelty in

283 itself (Réale et al., 2007; Edwards et al., 2013), or alternatively become less responsive or sensitized (Budaev,

284 1997; Martin \& Réale, 2008; Kelley, Phillips \& Evans, 2013). In our study, the tests that involved an

285 experimental set-up, such as laboratory-based measurements of boldness (e.g. latency to emerge), have some

286 of the largest confident intervals. However, given our significant repeatability estimates, we are confident all

287 the measures reported are reliable measures of an individual's behavior within these contexts.

288 Variables that originated from the aggression assay (strike latency and mirror strike rate) were the only

289 measurements found not to be repeatable through time or context. This suggests the moderate negative

290 correlation found between field and laboratory measures of aggression strike latency is likely to be ecologically

291 irrelevant. While a commonly-used test (Gerlai, 2003; Marks et al., 2005), these measures may be susceptible

292 to the habituation effect discussed above. A closely-related species, P. moluccensis, has been shown to

293 recognize threats after a single exposure (Mitchell et al., 2011). Perhaps P. amboinensis similarly learns to

294 ignore the false threat of their reflection after repeated exposures.

295 Observations repeated over short time scales (4 min apart, simultaneous video vs. video observations) had the

296 highest repeatability scores. Measures conducted over longer (30 minutes apart and 3 times daily over 3 days)

297 time periods had similar, but lower scores. This agrees with results from a meta-analysis, which showed higher

298 estimates of repeatability for behaviors measured at shorter time intervals (Bell, Hankison \& Laskowski, 2009).

299 Our results suggest juvenile damselfish quickly adopt stable behavioral phenotypes regarding foraging and

300 activity rates following settlement and remain consistent throughout the intense predation pressure

301 experienced during the first few days on the reef.

302 There was a trend for repeatability estimates obtained in the laboratory to be lower compared to field-based

303 measurements. This same pattern was found in Bell et al.'s (2009) meta-analysis. If there are advantages to 
304 behaving consistently (Dall, Houston \& McNamara, 2004; McElreath \& Strimling, 2006), then the greater

305 environmental variance in the field might create micro-niches, increasing repeatability by allowing individual

306 expression of behavioral variations (Bell, Hankison \& Laskowski, 2009). Also, because juveniles are exposed to

307 innately higher predation pressure in the field, this could act as a directional or stabilizing selection on behavior

308 (Bell, Hankison \& Laskowski, 2009). However, in this study fish are initially naïve and neophobic upon

309 introduction to the field (Meekan et al., 2010; Chivers et al., 2014; Ferrari et al., 2015), so perhaps the greater

310 sensory input in the field environment is enough to act as a stabilizing influence. A recent study found three-

311 spined sticklebacks (Gasterosteus aculeatus) adopted stable boldness-aggressiveness correlations once

312 exposed to predators (Bell \& Sih, 2007). Juvenile damselfish quickly learn about predators (Mitchell et al.,

313 2011) and are likely to swiftly adopt a consistent behavioral phenotype when faced with the variations and

314 challenges of their natural habitat. Given the few and weak correlations found between field and laboratory

315 measures, and lower consistency for laboratory studies suggests inferences about natural behaviors in the field

316 derived from laboratory studies need to be made cautiously (White, McCormick \& Meekan, 2013). The lack of

317 predators and increased novelty of the laboratory environment may enable juvenile damselfish to exhibit a

318 great variability of behaviors or prompt different behavioral responses that have little bearing on likely

319 behavior under natural conditions. This implies behavioral studies have limited predictive ability across

320 situations, in particular using laboratory measures to predict behaviors in the field (White et al., 2013; White,

321 McCormick \& Meekan, 2013).

322 Interestingly, Beckmann and Biro (2013) reported repeatability values almost identical to ours for the same

323 laboratory-based boldness measure. They tested two species of juvenile damselfish ( $P$. wardi and $P$.

324 amboinensis) and showed repeatability in the emergence latency test in home tanks ( $\mathrm{R}=0.42$ for $P$.

325 amboinensis on the third observation), but no correlations when compared against the same and different

326 behavioral tests in different contexts. Others have also argued for the use of multiple measures of boldness in

327 order to obtain an ecologically relevant assessment of this behavioral trait (White et al., 2013), and have also 
328 found a lack of behavioral consistency across situations (White, McCormick \& Meekan, 2013) for juvenile

329 damselfish. While Beckmann and Biro (2013) argue the lack of correlation across contexts means this assay is

330 inadequate to measure boldness, their study likely had issues with habituation (Edwards et al., 2013). In

331 contrast, we found latency to emerge behavior to be significantly repeatable within a single context and

332 moderately positively correlated with an emergence test in the field.

333 Another important result of our study was that the presence of observers seemed to have no significant impact

334 on fish behavior. While fishes are the focus of much behavioral research, they are rarely observed in their

335 natural environments (Réale et al., 2000; Bell, Hankison \& Laskowski, 2009). Typically, observations in a field

336 situation would be conducted from behind a blind (Martin \& Bateson, 2007), a luxury not afforded to a noisy

337 bubble-blowing SCUBA diver. While the simultaneous observer and video observations had slightly lower

338 repeatability scores for bite rate and height compared to the comparison of the two video scores (difference of

3390.23 and 0.13 , respectively), this is most likely an artifact of the difficulties associated with observing detailed

340 behavior via camera. Even with high resolution video, it was difficult to distinguish between feeding strikes and

341 the natural stop-start swimming of these fish. Also, fish leaving the field of view of the camera for a short

342 duration was not an issue for the diver who could maintain visual contact with the target fish at all times.

343 Overall, discrepancies between the methods of observation may have resulted in a slight over-counting of bite

344 rates in the video. This suggests video data is less useful for subjects such as these small damselfish that are

345 quick moving and very mobile. As long as slow, deliberate movements are employed and the observer remains

346 a least a meter away, juvenile damselfish seem indifferent to human presence thus diver observations provide

347 useful records of behavior.

348 In summary, our results demonstrate that measures of boldness and activity, both in the field and the

349 laboratory, are highly repeatable over time scales relevant to this species during a key period of their life

350 history. These stable behaviors indicate that these 3-week old juvenile fish already have personalities. From a

351 methodological perspective, our results indicate that an initial 3 min assessment of their behavior provides a 
352 useful record of an individual's personality. However, caution is required when comparing field and laboratory

353 based behaviors (White et al., 2013). Future studies with this species can reasonably use a single (i.e.

354 unrepeated) assay to reduce animal stress, which can then be correlated with physical measures of

355 performance and success to determine how individual characteristics combine to affect fitness. Future

356 research will investigate if adult $P$. amboinensis retain this behavioral consistency through ontogeny. 


\section{Acknowledgements}

359 We would like to thank C. Mero for collecting some of the initial data associated with the project. S. Banana

360 provided guidance and support throughout the study. J. Smart kindly assisted in the field and we thank the 361 staff of Lizard Island Research Station (Australia Museum) for generous logistical support. 
363

364 365

366

\section{References}

Adolph S, Hardin J. 2007. Estimating phenotypic correlations: Correcting for bias due to intraindividual variability. Functional Ecology 21:178-184.

Almany GR, Webster MS. 2006. The predation gauntlet: early post-settlement mortality in reef fishes. Coral Reefs 25:19-22.

Beckmann C, Biro PA. 2013. On the Validity of a Single (Boldness) Assay in Personality Research. Ethology:1-11.

Bell AM. 2005. Behavioural differences between individuals and two populations of stickleback (Gasterosteus aculeatus). Journal of Evolutionary Biology 18:464-473.

Bell AM. 2007. Future directions in behavioural syndromes research. Proceedings of the Royal Society B: Biological Sciences 274:755-761.

Bell AM, Hankison SJ, Laskowski KL. 2009. The repeatability of behaviour: a meta-analysis. Animal Behaviour 77:771-783.

Bell AM, Sih A. 2007. Exposure to predation generates personality in threespined sticklebacks (Gasterosteus aculeatus). Ecology Letters 10:828-834.

Bergmuller R, Schurch R, Hamilton IM. 2010. Evolutionary causes and consequences of consistent individual variation in cooperative behaviour. Philosophical Transactions of the Royal Society B: Biological Sciences 365:2751-2764.

Beukers J, Jones G. 1998. Habitat complexity modifies the impact of piscivores on a coral reef fish population. Oecologia 114:50-59.

Biro PA, Post JR. 2008. Rapid depletion of genotypes with fast growth and bold personality traits from harvested fish populations. Proceedings of the National Academy of Sciences 105:2919 -2922. 
385 Boake CR. 1994. Quantitative Genetic Studies of Behavioral Evolution. Chicago: University of $386 \quad$ Chicago Press.

387 Both C, Dingemanse NJ, Drent PJ, Tinbergen JM. 2005. Pairs of extreme avian personalities have 388 highest reproductive success. Journal of Animal Ecology 74:667-674.

389 Briffa M, Greenaway J. 2011. High In Situ Repeatability of Behaviour Indicates Animal Personality in 390 the Beadlet Anemone Actinia equina (Cnidaria). PLoS ONE 6:e21963.

Brown C, Jones F, Braithwaite V. 2005. In situ examination of boldness-shyness traits in the tropical poeciliid, Brachyraphis episcopi. Animal Behaviour 70:1003-1009.

Brunton B, Booth D. 2003. Density- and size-dependent mortality of a settling coral-reef damselfish (Pomacentrus moluccensis Bleeker). Oecologia 137:377-384.

Budaev SV. 1997. "Personality" in the guppy (Poecilia reticulata): A correlational study of exploratory behavior and social tendency. Journal of Comparative Psychology 111:399-411.

Burtka JL, Grindstaff JL. 2013. Repeatable nest defense behavior in a wild population of Eastern bluebirds (Sialia sialis) as evidence of personality. acta ethologica 16:135-146.

Carter AJ, Marshall HH, Heinsohn R, Cowlishaw G. 2012. How not to measure boldness: novel object and antipredator responses are not the same in wild baboons. Animal Behaviour 84:603-609.

Chang C, Li CY, Earley RL, Hsu Y. 2012. Aggression and related behavioral traits: the impact of winning and losing and the role of hormones. Integrative and Comparative Biology.

Chivers DP, McCormick MI, Mitchell MD, Ramasamy RA, Ferrari MCO. 2014. Background level of risk determines how prey categorize predators and non-predators. Proceedings of the Royal Society B: Biological Sciences 281:20140355-20140355.

Couchoux C, Cresswell W. 2012. Personality constraints versus flexible antipredation behaviors: how important is boldness in risk management of redshanks (Tringa totanus) foraging in a natural system? Behavioral Ecology 23:290-301. 
409 Dall SRX, Houston AI, McNamara JM. 2004. The behavioural ecology of personality: consistent $410 \quad$ individual differences from an adaptive perspective. Ecology Letters 7:734-739.

411 DeWitt TJ, Sih A, Wilson DS. 1998. Costs and limits of phenotypic plasticity. Trends in Ecology and Evolution 13:77-81.

413 Dingemanse NJ, Both C, Drent PJ, Tinbergen JM. 2004. Fitness consequences of avian personalities in 414 a fluctuating environment. Proceedings of the Royal Society of London. Series B: Biological Sciences 271:847-852.

Dingemanse NJ, Van der Plas F, Wright J, Réale D, Schrama M, Roff DA, Van der Zee E, Barber I. 2009. Individual experience and evolutionary history of predation affect expression of heritable variation in fish personality and morphology. Proceedings of the Royal Society B: Biological Sciences 276:1285.

Dingemanse NJ, Kazem AJN, Réale D, Wright J. 2010. Behavioural reaction norms: animal personality meets individual plasticity. Trends in Ecology \& Evolution 25:81-89.

Dingemanse NJ, Réale D. 2005. Natural selection and animal personality. Behaviour 142:1159-1184.

Doherty PJ, Dufour V, Galzin R, Hixon MA, Meekan MG, Planes S. 2004. High mortality during settlement is a population bottleneck for a tropical surgeonfish. Ecology 85:2422-2428.

Donner A, Wells G. 1986. A Comparison of Confidence Interval Methods for the Intraclass Correlation Coefficient. Biometrics 42:401.

Downes SJ. 2002. Does responsiveness to predator scents affect lizard survivorship? Behavioural Ecology and Sociobiology 52:38-42.

Edwards HA, Winney IS, Schroeder J, Dugdale HL. 2013. Do rapid assays predict repeatability in labile (behavioural) traits? A reply to Biro. Animal Behaviour 85:e1-e3. 
431 Ferrari MCO, McCormick MI, Meekan MG, Chivers DP. 2015. Background level of risk and the

432 survival of predator-naive prey: can neophobia compensate for predator naivety in juvenile coral reef fishes? Proceedings of the Royal Society B: Biological Sciences.

Fraser DF, Gilliam JF, Daley MJ, Le AN, Skalski GT. 2001. Explaining leptokurtic movement distributions: intrapopulation variation in boldness and exploration. American Naturalist 158:124-135.

Garamszegi LZ, Herczeg G. 2012. Behavioural syndromes, syndrome deviation and the within- and between-individual components of phenotypic correlations: when reality does not meet statistics. Behavioral Ecology and Sociobiology 66:1651-1658.

Gerlai R. 2003. Zebrafish: an uncharted behavior genetic model. Behavior Genetics 33:461-468.

Johnson J, Sih A. 2005. Pre-coupulatory sexual cannibalism in fishing spiders (Dolomedes triton): A role for behavioral syndromes. Behavioral Ecology and Sociobiology 58:390-396.

Jones KA, Godin J-GJ. 2009. Are fast explorers slow reactors? Linking personality type and antipredator behaviour. Proceedings of the Royal Society B: Biological Sciences 277:625-632.

Kelley JL, Phillips SC, Evans JP. 2013. Individual consistency in exploratory behaviour and mating tactics in male guppies. Naturwissenschaften.

Kolliker M. 2005. Ontogeny in the family. Behavior Genetics 35:7-18.

Lessells CM, Boag PT. 1987. Unrepeatable repeatabilities: a common mistake. The Auk:116-121.

Marks C, West T, Bagatto B, Moore F. 2005. Developmental environment alters conditional aggression in zebrafish. Copeia:901-908.

Marras S, Killen SS, Claireaux G, Domenici P, McKenzie DJ. 2011. Behavioural and kinematic components of the fast-start escape response in fish: individual variation and temporal repeatability. Journal of Experimental Biology 214:3102-3110. 
454 Martin P, Bateson PPG. 2007. Measuring Behaviour: An Introductory Guide. Cambridge University

$455 \quad$ Press.

456 Martin JGA, Réale D. 2008. Temperment, risk assessment and habitatuation to novelty in eastern 457 chipmunks, Tamias striatus. Animal Behaviour 75:309-318.

458

459

460

461

462

463

464

465

466

467

468

469

470

471

472

473

474

475

476

McCormick MI, Makey LJ. 1997. Post-settlement transition in coral reef fishes: overlooked complexity in niche shifts. Marine Ecology Progress Series 153:247-257.

McCormick MI, Meekan MG. 2010. The importance of attitude: the influence of behaviour on survival at an ontogenetic boundary. Marine Ecology Progress Series 407:173-185.

McCormick MI, Weaver CJ. 2012. It Pays to Be Pushy: Intracohort Interference Competition between Two Reef Fishes. PLOS ONE 7.

McElreath R, Strimling P. 2006. How noisy in formation and individual asymmetries can make “personality” an adaptation: a simple model. Animal Behaviour 72:1135-1139.

Meekan MG, Wilson SG, Halford A, Retzel A. 2001. A comparison of catches of fishes and invertebrates by two light trap designs, in tropical NW Australia. Marine Biology 139:373-381.

Meekan MG, von Kuerthy C, McCormick MI, Radford B. 2010. Behavioural mediation of the costs and benefits of fast growth in a marine fish. Animal Behaviour 79:803-809.

Meekan MG, Milicich MJ, Doherty PJ. 1993. Larval production drives temporal patterns of larval supply and recruitment of a coral reef damselfish. Marine Ecology Progress Series 93:217-225.

Mitchell MD, McCormick MI, Ferrari MCO, Chivers DP. 2011. Friend or foe? The role of latent inhibition in predator and non-predator labelling by coral reef fishes. Animal Cognition 14:707714.

Mittelbach GG, Ballew NG, Kjelvik MK, Fraser D. 2014. Fish behavioral types and their ecological consequences. Canadian Journal of Fisheries and Aquatic Sciences 71:927-944. 
Nakagawa S, Gillespie DOS, Hatchwell BJ, Burke T. 2007. Predictable males and unpredictable females: sex difference in repeatability of parental care in a wild bird population: Sex difference in repeatability of parental care. Journal of Evolutionary Biology 20:1674-1681.

Neumann C, Agil M, Widdig A, Engelhardt A. 2013. Personality of Wild Male Crested Macaques (Macaca nigra). PloS one 8:e69383.

Van Oers K, de Jong G, van Noordwijk AJ, Kempenaers B, Drent PJ. 2005. Contribution of genetics to the study of animal personalities: a review of case studies. Behaviour 142:1185-1206.

Penke L, Denissen JJA, Miller GF. 2007. The Evolutionary Genetics of Personality. European Journal of Personality 21:549-587.

Pitcher CR. 1988. Validation of a technique for reconstructing daily patterns in the recruitment of coral reef damselfish. Coral Reefs 7:105-111.

Poulos DE, McCormick MI. 2014. Who wins in the battle for space? The importance of priority, behavioural history and size. Animal Behaviour 90:305-314.

Pruitt JN, Ferrari MCO. 2011. Intraspecific trait variants determine the nature of interspecific interactions in habitat forming species. Ecology 92:1902-1908.

Pruitt JN, Grinsted L, Settepani V. 2013. Linking levels of personality: personalities of the "average" and "most extreme" group members predict colony-level personality. Animal Behaviour 86:391-399.

Réale D, Gallant BY, Leblanc M, Festa-Bianchet M. 2000. Consistency of temperment in bighorn ewes and correlates with behaviour and life history. Animal Behaviour 60:589-597.

Réale D, Reader SM, Sol D, McDougall PT, Dingemanse NJ. 2007. Integrating animal temperament within ecology and evolution. Biological Reviews 82:291-318.

Riechert SE, Hedrick AV. 1993. A test for correlations among fitness-linked behavioral traits in the spider Agelenopsis aperta (Araneae, Agelenidae). Animal Behaviour 46:669-675. 
501 Searle SR. 1971. Linear Models. New York: Wiley.

502 Sih A, Bell AM, Johnson JC, Ziemba RE. 2004. Behavioral syndromes: an integrative overview.

$503 \quad$ Quarterly Review of Biology 79:241-277.

504 Sih A, Cote J, Evans M, Fogarty S, Pruitt JN. 2012. Ecological implications of behavioural syndromes.

$505 \quad$ Ecology Letters 15:278-289.

506 Sih A, Bell AM. 2008. Insights for Behavioral Ecology from Behavioral Syndromes. Advances in the $507 \quad$ Study of Behavior 38:227-281.

508 Sih A, Bell AM, Johnson JC. 2004. Behavioral syndromes: an ecological and evolutionary overview. $509 \quad$ Trends in Ecology \& Evolution 19:372-378.

510 Sih A, Watters JV. 2005. The mix matters: behavioural types and group dynamics in water striders. $511 \quad$ Behaviour 142:1417-1431.

512 Smith BR, Blumstein DT. 2008. Fitness consequences of personality: a meta-analysis. Behavioral $513 \quad$ Ecology 19:448-455.

514 Stirling DG, Reale D, Roff DA. 2002. Selection, structure and the heritability of behaviour. Journal of $515 \quad$ Evolutionary Biology 15:277-289.

516 Webster MM, Ward AJW, Hart PJB. 2009. Individual boldness affects interspecific interactions in sticklebacks. Behavioural Ecology and Sociobiology 63:511-520.

518 Wellington G, Victor B. 1989. Planktonic larval duration of one hundred species of Pacific and $519 \quad$ Atlantic damselfishes (Pomacentridae). Marine Biology 101:557-567.

520 White JR, Meekan MG, McCormick MI, Ferrari MCO. 2013. A comparison of measures of boldness 521 and their relationships to survival in young fish. PLOS ONE 8.

522 White JR, McCormick MI, Meekan MG. 2013. Syndromes or Flexibility: Behavior during a Life 523 History Transition of a Coral Reef Fish. PLoS ONE 8:e84262. 
524 Wolak ME, Fairbairn DJ, Paulsen YR. 2012. Guidelines for estimating repeatability. Methods in 525 Ecology and Evolution 3:129-137.

526 


\section{Table $\mathbf{1}_{\text {(on next page) }}$}

Table 1: Repeatability ( $\mathrm{R}$ ) values with $95 \%$ confidence intervals $(\mathrm{Cl})$ for various measures of boldness and activity for juvenile Ambon Damselfish (Pomacentrus amboinensis).

For the observer vs. video section, the human observation is labeled 'observer', the simultaneous video camera recording 'simultaneous video', and the independent video recording 'video'. $\dagger=$ Individual reaction norm graph available in supplementary materials. 


\begin{tabular}{|c|c|c|c|c|}
\hline Trait & $\mathbf{R}$ & $\mathbf{p}$ & R Cl Low & R Cl High \\
\hline \multicolumn{5}{|l|}{ Field } \\
\hline \multicolumn{5}{|c|}{ Short term consistency $(n=18)$} \\
\hline Bite rate & 0.64 & $<0.001$ & 0.39 & 0.83 \\
\hline Distance ventured $\dagger$ & 0.69 & $<0.001$ & 0.46 & 0.86 \\
\hline Reef height & 0.52 & $<0.001$ & 0.24 & 0.76 \\
\hline Aggression latency & 0.20 & NS & 0.07 & 0.52 \\
\hline Aggression strikes & 0.20 & NS & 0.07 & 0.52 \\
\hline \multicolumn{5}{|l|}{ Multiple days $(n=21)$} \\
\hline Bite rate ${ }^{\dagger}$ & 0.77 & $<0.001$ & 0.64 & 0.88 \\
\hline Distance ventured & 0.62 & $<0.001$ & 0.45 & 0.79 \\
\hline Reef height & 0.33 & $<0.001$ & 0.16 & 0.55 \\
\hline \multicolumn{5}{|c|}{ Observer vs. video ( $n=29)$} \\
\hline \multicolumn{5}{|c|}{ Observer vs. simultaneous video } \\
\hline Bite rate & 0.46 & 0.005 & 0.13 & 0.71 \\
\hline Reef height & 0.76 & $<0.001$ & 0.56 & 0.88 \\
\hline \multicolumn{5}{|c|}{ Simultaneous video vs. video } \\
\hline Bite rate & 0.69 & $<0.001$ & 0.45 & 0.84 \\
\hline Reef height ${ }^{\dagger}$ & 0.89 & $<0.001$ & 0.79 & 0.95 \\
\hline \multicolumn{5}{|c|}{$\begin{array}{l}\text { Laboratory } \\
\text { Short term consistency }(n=10)\end{array}$} \\
\hline Latency to emerge ${ }^{\dagger}$ & 0.38 & 0.026 & -0.004 & 0.76 \\
\hline Location & 0.54 & 0.003 & 0.16 & 0.84 \\
\hline
\end{tabular}


Table 2 (on next page)

Table 2: Pearson's product-moment correlations between field and laboratory measures of boldness and aggression for juvenile Ambon damselfish (Pomacentrus amboinensis).

All data $(n=32)$ was $\log (x=1)$ transformed. 


\begin{tabular}{c|cccccc} 
Trait & Field L & Field BR & Field DV & Field height & Field AL & Field ASR \\
\hline Lab L & $0.350^{*}$ & 0.169 & 0.110 & -0.102 & 0.027 & 0.202 \\
Lab Location & 0.189 & 0.094 & -0.147 & 0.044 & 0.156 & -0.272 \\
Lab AL & 0.144 & -0.079 & 0.067 & 0.227 & $-0.385^{*}$ & -0.090 \\
Lab ASR & -0.088 & -0.051 & -0.016 & 0.172 & -0.262 & -0.037
\end{tabular}

2 Note. $\mathrm{L}=$ latency, $\mathrm{BR}=$ Bite rate, $\mathrm{DV}=$ Distance ventured, $\mathrm{H}=$ Height, $\mathrm{AL}=$ Aggression latency, $\mathrm{ASR}=$ Aggression 3 strike rate, $*=$ statistically significant at $p<0.05$ level 